\title{
Affinity Cryo-Electron Microscopy Studies of Viral Particles Captured Directly From Cell Culture
}

\author{
Ci Zhang ${ }^{1 *}$, Frank Vago ${ }^{1 *}$, Fei Guo ${ }^{1}$, Zheng Liu ${ }^{1}$, Guimei Yu ${ }^{1}$, Phil Serwer ${ }^{2}$, and Wen Jiang ${ }^{1}$ \\ ${ }^{1}$ Markey Center for Structural Biology, Department of Biological Sciences, Purdue University, West \\ Lafayette, IN 47907 \\ ${ }^{2}$ Department of Biochemistry, University of Texas Health Science Center, San Antonio, TX 78229 \\ *Equal contribution
}

Single particle cryo-electron microscopy (cryo-EM) and 3-D reconstruction have emerged in recent years as a powerful tool for solving high-resolution 3-D structures of viruses and macromolecular complexes. However, sample preparation for single-particle cryo-EM typically relies on a dedicated purification step before EM sample grid preparation, for example, using column chromatography or gradient centrifugation, to obtain homogeneous samples at high concentration. This strategy usually uses large volume of cell culture (hundreds of milli-liters to multiple liters) and takes hours to days to complete. While stable major states of the sample can be successfully prepared, many of the short-lived intermediate states won't be able to survive the lengthy duration and those minor low abundance states are prone to being ignored. For example, until our attempt with T3 phage [1], tailed dsDNA phage intermediates with incompletely packaged dsDNA genome eluded cryo-EM investigation due to their instability and the faint band visibility in the gradient centrifugation due to low abundance. Therefore, improved sample preparation methods, for example, the affinity cryo-EM grids [2-5], are needed to allow sampling of all states, including the minor, transient intermediates, to fully cover the complete process of the targets for better understanding of structures and functions. Four different affinity grid techniques have been developed for single-particle cryo-EM sample preparation: the Ni-NTA lipid monolayer method [5], the streptavidin 2D crystal-based method [4], the functionalized carbon film method [3], and single-step antibody-based method [2]. However, no structures of subnanometer or higher resolutions using these affinity-grid approaches have been reported and there are concerns if the affinity grids will limit the reconstructions to low resolutions.

Here we employed the Ni-NTA lipid monolayer based affinity grid to capture His-tagged T7 phage particles directly from small volume of bacterial lysate for single-particle cryo-EM imaging and 3-D reconstruction. We used the T7Select ${ }^{\circledR}$ phage display system (Merck Chemicals) to construct a new T7 phage variant with 6x His-tag at the C-terminus of all 415 copies of major capsid protein gp10 in the phage particles. Plaque assays showed that the new T7His phage has normal infectivity as wild type T7 phage. Lipid monolayer based affinity EM grids were prepared using a 10:1 weight ratio of DOPC and Ni-NTA lipid. To maximally capture all possible T7 particle states including low abundance or shortlived intermediate states, Ni-NTA affinity grids were soaked with cell lysate immediately after the cells being lysed by the inoculated T7 phage. Cryo-EM was then used to gather a large set of micrographs of the captured particles using the Titan Krios microscope at low dose conditions.

From the micrographs, we could observe a large number of particles of various sizes, shapes, and aggregation states (Figure 1). Most of the particles can be visually classified into the three major states of T7 capsids, capsid I, capsid II, and mature phage, which have been thoroughly characterized in past cryo-EM studies using particles purified with traditional centrifugation method [6]. Many additional unusual states are also observed, for example, tubes, prolate particles, "Pac-man"-like particles, 
paracrystalline-like fiber clusters, and lipid wrapped particles. The three major states, capsid I, capsid II, and mature phage, of T7 phage particles represent three different steps in the T7 maturation [6]. We could determine the icosahedral structure of all three states to subnanometers resolutions of capsid I at $6.9 \AA$ resolution, capsid II at $8.1 \AA$ resolution, and mature phage at $7 \AA$ resolution, respectively, based on "gold" standard Fourier Shell Correlation criterion (Figure. 2). From the icosahedral shell densities of all three structures, $\alpha$-helices and $\beta$-sheets can be identified as rod-like and curved flat shape features, respectively, and the HK97-like protein fold [7] can also be readily recognized. Our results suggest that affinity cryo-EM would be a general approach suitable for imaging and high resolution structural studies of the dynamics process of a wide range of biological systems [8].

\section{References:}

[1] PA Fang, ET Wright, ST Weintraub et al. Visualization of bacteriophage T3 capsids with DNA incompletely packaged in vivo. J Mol Biol 384 (2008), p. 1384-1399

[2] G Yu, F Vago, D Zhang et al. Single-step antibody-based affinity cryo-electron microscopy for imaging and structural analysis of macromolecular assemblies. J Struct Biol 187 (2014), p. 1-9

[3] MC Llaguno, $\mathrm{H} \mathrm{Xu}, \mathrm{L}$ Shi et al. Chemically functionalized carbon films for single molecule imaging. J Struct Biol 185 (2014), p. 405-417

[4] BG Han, RW Walton, A Song et al. Electron microscopy of biotinylated protein complexes bound to streptavidin monolayer crystals. J Struct Biol 180 (2012), p. 249-253

[5] DF Kelly, D Dukovski \& T Walz Monolayer purification: a rapid method for isolating protein complexes for single-particle electron microscopy. Proc Natl Acad Sci U S A 105 (2008), p. 47034708

[6] F Guo, Z Liu, P Fang et al. Capsid expansion mechanism of bacteriophage T7 revealed by multistate atomic models derived from cryo-EM reconstructions. Proceedings of the National Academy of Sciences of the United States of America 111 (2014), p. E4606-14

[7] WR Wikoff, L Liljas, RL Duda et al. Topologically linked protein rings in the bacteriophage HK97 capsid. Science 289 (2000), p. 2129-2133

[8] The authors acknowledge funding from the National Institutes of Health and Welch Foundation. The cryo-EM images were taken in the Purdue Cryo-EM Facility, and the Purdue Rosen Center for Advanced Computing provided the computational resource for the $3 \mathrm{D}$ reconstructions.

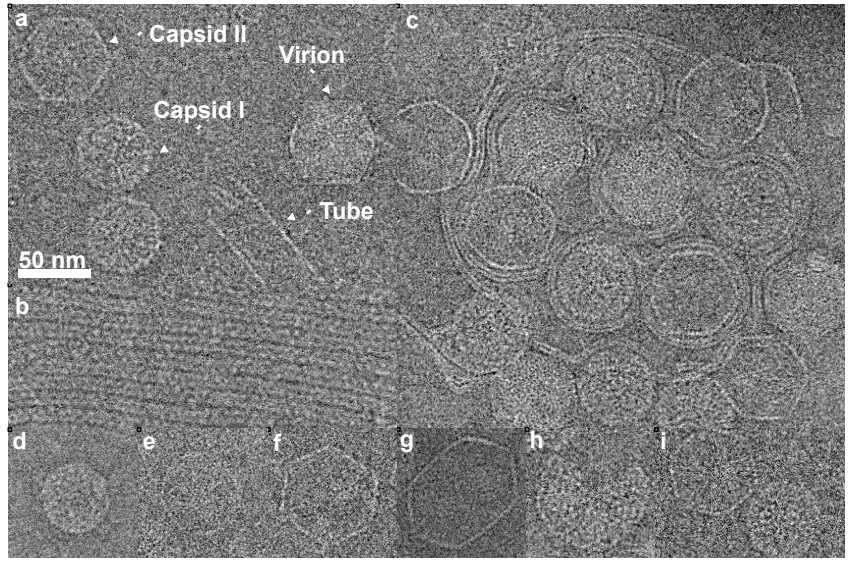

Figure 1. Gallery of T7His phage particles affinity-captured from $E$. coli lysate.

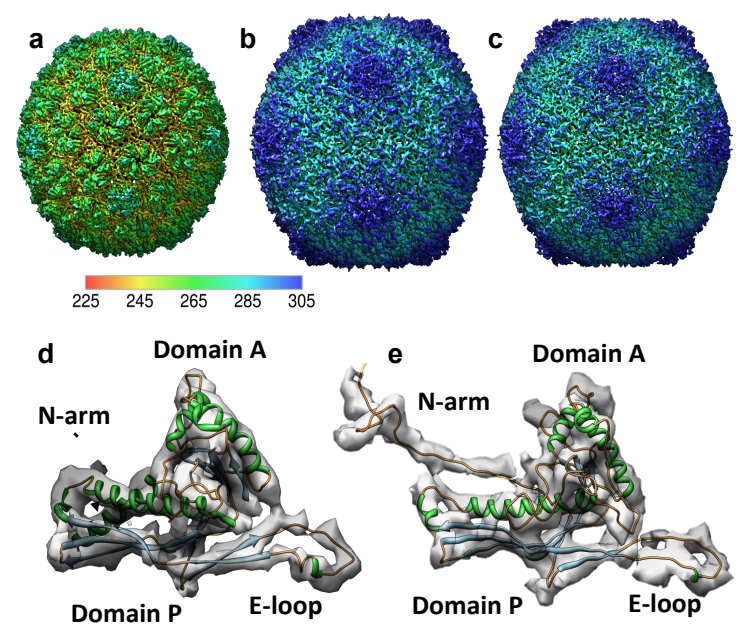

Figure 2. 3-D reconstructions of capsid I, capsid II and virion affinity-captured from lysate. 